\title{
Liver Fibrosis Indices Associated with Substantial Hematoma Expansion in Chinese Patients with Primary Intracerebral Hemorrhage
}

\section{Huan Wang}

West China Hospital of Sichuan University

Jiongxing Wu

West China Hospital of Sichuan University

\section{Xue Yang}

West China Hospital of Sichuan University

Junfeng Liu

West China Hospital of Sichuan University

Wendan Tao

West China Hospital of Sichuan University

\section{Zilong Hao}

West China Hospital of Sichuan University

\section{Bo Wu}

West China Hospital of Sichuan University

Ming Liu

West China Hospital of Sichuan University

\section{Shihong Zhang}

West China Hospital of Sichuan University

Deren Wang ( $\square$ wangderen@wchscu.cn )

West China Hospital of Sichuan University

\section{Research Article}

Keywords: hemorrhagic stroke, liver fibrosis indices, hematoma enlargement, AST-platelet ratio index, fibrosis-4

Posted Date: July 7th, 2021

DOl: https://doi.org/10.21203/rs.3.rs-666991/v1

License: (c) (1) This work is licensed under a Creative Commons Attribution 4.0 International License.

Read Full License 
Version of Record: A version of this preprint was published at BMC Neurology on December 1st, 2021. See the published version at https://doi.org/10.1186/s12883-021-02494-0. 


\section{Liver Fibrosis Indices Associated with Substantial Hematoma Expansion in Chinese Patients with Primary Intracerebral Hemorrhage}

Huan Wang ${ }^{1}$, Jiongxing $\mathrm{Wu}^{1}$, Xue Yang ${ }^{1}$, Junfeng $\mathrm{Liu}^{1}$, Wendan $\mathrm{Tao}^{1}$, Zilong Hao ${ }^{1}$, Bo $\mathrm{Wu}^{1}$, Ming Liu ${ }^{1}$, Shihong Zhang ${ }^{1 *}$, Deren Wang ${ }^{1^{*}}$

${ }^{1}$ Department of Neurology, Center of Cerebrovascular Disease, West China Hospital, Sichuan University, No. 37 Guo Xue Xiang, Chengdu, 610041, Sichuan Province, P.R. China

*Correspondence: Deren Wang, MD, PhD, Department of Neurology, Center of Cerebrovascular Disease, West China Hospital, Sichuan University, No. 37 Guo Xue Xiang, Chengdu, 610041, China, E-mail: wangderen@wchscu.cn; Shihong Zhang, $\mathrm{MD}, \mathrm{PhD}$, Department of Neurology, Center of Cerebrovascular Disease, West China Hospital, Sichuan University, No. 37 Guo Xue Xiang, Chengdu, 610041, China, E-mail: zsh9@hotmail.com.

Running title: Substantial hematoma expansion and liver fibrosis 


\section{Abstract}

Background: Whether liver fibrosis is associated with increased risk for substantial hematoma expansion (HE) after intracerebral hemorrhage (ICH) is still uncertain. We evaluated the association between various liver fibrosis indices and substantial HE in a Chinese population with primary ICH.

Methods: Primary ICH patients admitted to West China Hospital within 24 hours of onset between January 2015 and June 2018 were consecutively enrolled. Six liver fibrosis indices were calculated, including aspartate aminotransferase (AST)-platelet ratio index (APRI), AST/alanine aminotransferase ratio-platelet ratio index (AARPRI), fibrosis-4 (FIB-4), modified fibrosis-4 (mFIB-4), fibrosis quotient (FibroQ) and Forns index. Substantial HE was defined as an increase of more than $33 \%$ or $6 \mathrm{~mL}$ from baseline ICH volume. The association of each fibrosis index with substantial HE was analyzed using binary logistic regression.

Results: Of 436 patients enrolled, about $85 \%$ showed largely normal results on standard hepatic assays and coagulation parameters. Substantial HE occurred in 115 (26.4\%) patients. After adjustment, AARPRI (OR 1.26, 95\% CI 1.00-1.57) and FIB-4 (OR 1.15, 95\% CI 1.02-1.30) were independently associated with substantial HE in ICH patients within $24 \mathrm{~h}$ of onset, respectively. In ICH patients within $6 \mathrm{~h}$ of onset, each of the following indices was independently associated with substantial HE: APRI (OR 2.63, 95\% CI 1.30-5,34), AARPRI (OR 1.55, 95\% CI 1.09-2.22), FIB-4 (OR 1.35, 95\% CI 1.08-1.69), mFIB-4 (OR 1.09, 95\% CI 1.01-1.18), FibroQ (OR 1.08, 95\% CI 1.00-1.16) and Forns index (OR 1.37, 95\% CI 1.10-1.69). 
Conclusions: Liver fibrosis indices are independently associated with higher risk of substantial HE in Chinese patients with primary ICH, which suggesting that subclinical liver fibrosis could be routinely assessed in such patients to identify those at high risk of substantial HE.

Keywords: hemorrhagic stroke; liver fibrosis indices; hematoma enlargement; ASTplatelet ratio index; fibrosis-4 


\section{Introduction}

Intracerebral hemorrhage (ICH) accounts for $10 \%$ to $15 \%$ of all strokes, and causes up to $50 \%$ of all stroke-related mortality. ${ }^{1-3}$ More than one-third of patients with ICH experience hematoma expansion (HE), which is a strong predictor of early deterioration and death. ${ }^{4,5}$ However, specific risk factors for HE and the impact of the heterogeneity of ICH on HE are unclear. ${ }^{6}$ To date, randomized controlled trials of several interventions targeting HE have not demonstrated any improvement in outcomes after ICH. ${ }^{7,8}$ Current paradigms of treatment after ICH focus mainly on coagulopathy reversal in order to minimize HE and improve survival and functional outcomes. ${ }^{3,9}$ Therefore, understanding the risk factors of $\mathrm{HE}$ in patients with $\mathrm{ICH}$ may help to guide preventive measures and intervention post-ICH.

Increasing evidence links liver dysfunction to ICH. Patients with ICH and unusually large hematomas show elevated levels of liver enzymes, such as aspartate aminotransferase (AST), alanine aminotransferase (ALT) and gamma-glutamyl transferase (GGT). ${ }^{10} \mathrm{ICH}$ patients with comorbid liver disease are more likely to die inhospital and are in worse condition at discharge than those without liver disease. ${ }^{11}$ Even subclinical liver disease may be associated with worse prognosis post-ICH. ${ }^{12}$

Liver fibrosis is a common subclinical liver disease that is present in approximately $15 \%$ of the general adult population without diagnosed liver disease. ${ }^{13,14}$ Several noninvasive markers have been reported to predict liver fibrosis: AST/ALT ratio (AAR), AST- 
platelet ratio index (APRI), AAR/platelet ratio index (AARPRI), fibrosis-4 (FIB-4), modified fibrosis-4 (mFIB-4), fibrosis quotient (FibroQ) and the Forns index. ${ }^{15,16}$ AARPRI and mFIB-4, in particular, were developed to assess the stage of liver fibrosis in Asian patients with chronic viral hepatitis. ${ }^{17}$ These liver fibrosis indices allow simple, inexpensive, straightforward assessment of liver fibrosis, including in patients with viral hepatitis, non-alcoholic fatty liver disease (NAFLD) and alcohol-related liver disease (ALD). ${ }^{15-21}$

A recent study in patients with largely normal liver enzyme levels in a predominantly Caucasian population found that APRI and FIB-4 liver fibrosis indices were associated with $\mathrm{HE}$ post-ICH, linking $\mathrm{HE}$ to subclinical coagulopathy and endothelial dysfunction. ${ }^{22}$ However, there is little evidence on the relationship between liver fibrosis indices and HE in Asian patients without obvious liver diseases or significantly abnormal laboratory findings. Therefore, we aimed to evaluate the association between liver fibrosis indices and substantial HE after primary ICH in a Chinese population.

\section{Materials and Methods}

\section{Study design and cohort selection}

Patients with primary ICH who were admitted to West China Hospital within 24 hours of ICH symptom onset between January 2015 and June 2018 were retrospectively selected from among patients prospectively registered in our ICH database. ${ }^{23}$ The study was approved by the Ethics Committee on Biomedical Research, West China Hospital 
of Sichuan University (2019 362). No informed consent was required as this study was retrospective and all patient data were anonymized.

Patients were included if they received a baseline brain computed tomography (CT) scan immediately upon arrival in the Department of Emergency Medicine, as well as a follow-up brain CT scan within 72 hours after symptom onset. Patients were excluded if they met any of the following criteria: (1) were $<18$ years old; (2) had a secondary cause of ICH (i.e., underlying aneurysm, vascular malformation, brain neoplasm or metastasis, head trauma, dural sinus thrombosis, hemorrhagic transformation of ischemic infarction, infection), or primary intraventricular hemorrhage (IVH), or primary subarachnoid hemorrhage (SAH); (3) were on anticoagulant therapy before admission; (4) had severe liver disease, clinical syndrome associated with liver disease, or hematologic disease; (5) had undergone surgical evacuation; or (6) were lacking laboratory data of hepatic assays or coagulation parameters, which were tested routinely in most of patients.

\section{Data collection}

The following data were collected for all patients: demographic information including age, sex, Glasgow Coma Scale (GCS) score, National Institutes of Health Stroke Scale (NIHSS) score, as well as systolic and diastolic blood pressure on admission; comorbidities diagnosed either before admission or at discharge (hypertension, diabetes mellitus, hyperlipidemia, heart disease (includes any history of atrial fibrillation, 
coronary heart disease, or valvular heart disease); previous smoking (defined as smoking more than 10 cigarettes a day prior to onset, for at least 1 year) and alcohol consumption (defined as drinking almost every day, the average amount of daily drinking being more than $50 \mathrm{~g}$, for more than 1 year ); and the use of antiplatelets before admission. In addition, data were collected on the previous history of stroke (ischemic or hemorrhagic stroke).

Laboratory findings on admission included platelet count, international normalized ratio (INR), prothrombin time (PT), activated plasma thromboplastin time (APTT), ALT, AST and albumin (ALB). Data were also collected about onset of ICH symptoms, how long the patient was symptom-free, and when baseline and follow-up brain CT scans were performed.

The initial CT scan taken at presentation was reviewed to determine the ICH location (lobar, deep, and infratentorial), the presence of IVH, and baseline hematoma volumes. Hematoma volumes were estimated from $\mathrm{CT}$ scans using the formula $\mathrm{A}^{*} \mathrm{~B}^{*} \mathrm{C} / 2$, where A was the largest diameter on the largest hemorrhage slice; $\mathrm{B}$, the maximal diameter perpendicular to $\mathrm{A}$; and $\mathrm{C}$, the vertical hematoma depth. ${ }^{24}$ Intraventricular hemorrhage were not considered for volumetric calculations.

\section{Estimation of liver fibrosis indices}

We selected six liver fibrosis indices: APRI, AARPRI, FIB-4, mFIB-4, FibroQ, and Forns index. Fibrosis indices were calculated according to the following formulas ${ }^{16,17,21}$ : 


$$
\begin{gathered}
\text { APRI }=\frac{\text { AST }(/ \text { ULN })}{\text { Platelet count }\left(10^{9} / \mathrm{L}\right)} \times 100 \\
\text { AAR }=\frac{\text { AST }}{\text { ALT }} \text { ratio, AARPRI }=\frac{\text { AAR }}{\text { Platelet count }\left(10^{9} / \mathrm{L}\right) / 150} \\
\text { FIB }-4=\frac{\text { Age }(\text { years }) \times \text { AST }(\mathrm{U} / \mathrm{L})}{\text { Platelet count }\left(10^{9} / \mathrm{L}\right) \times \sqrt{\text { ALT }(\mathrm{U} / \mathrm{L})}} \\
\mathrm{mFIB}-4=\frac{10 \times \text { Age }(\text { years }) \times \text { AST }(\mathrm{U} / \mathrm{L})}{\text { Platelet count }\left(10^{9} / \mathrm{L}\right) \times \text { ALT }(\mathrm{U} / \mathrm{L})} \\
\text { FibroQ }=10 \times \frac{\text { Age }(\text { years }) \times \text { AST }(\mathrm{U} / \mathrm{L}) \times \mathrm{INR}}{\text { ALT }(\mathrm{U} / \mathrm{L}) \times \text { Platelet count }\left(10^{9} / \mathrm{L}\right)}
\end{gathered}
$$

Forns index $=7.811-3.131 \times \operatorname{In}\left(\right.$ Platelet $\left.\operatorname{count}\left[10^{9} / \mathrm{L}\right]\right)+0.781 \times \operatorname{In}(\gamma \mathrm{GT}[\mathrm{IU} / \mathrm{L}])$ $+3.467 \times \operatorname{In}($ age $)-0.014 \times$ cholesterol $(\mathrm{mg} / \mathrm{dl})$

\section{Outcome}

The outcome of interest was substantial HE within 72 hours of symptom onset. Substantial HE was defined as a proportional increase of hematoma volume $>33 \%$ or an absolute growth of hematoma volume $>6 \mathrm{~mL}$ from baseline CT scan to follow-up CT scan. ${ }^{25}$

\section{Statistical analysis}

Categorical variables were presented as $\mathrm{n}(\%)$, whereas continuous variables were presented as mean \pm standard deviation (SD) or median and interquartile range (IQR). 
Differences between groups with or without substantial HE were assessed for significance using the chi-squared test or Fisher exact test in the case of categorical variables, or using Student's t test or Mann-Whitney U test in the case of continuous variables.

Potential associations between liver fibrosis indices and substantial HE were estimated using binary logistic regression. All variables associated with $\mathrm{p} \leq 0.10$ were considered in the regression. Laboratory data were not included as variables in the regression, since the liver fibrosis indices were estimated from such data. To avoid effects of interactions between liver fibrosis indices, each index was included separately in the binary logistic regression. Since hematoma volume on admission may affect the association between liver fibrosis indices and substantial HE, we considered two models: Model 1 and Model 2, which contained the same variables as Model 1 in addition to baseline hematoma volume. Analysis was planned both in all patients and an early admission group within 6 hours of ICH onset.

Statistical analyses were conducted using SPSS 24.0 (IBM, New York, USA). Differences and statistical results associated with a 2-tailed $\mathrm{p}<0.05$ were defined as significant.

\section{Results}

\section{Baseline patient characteristics}


We identified 1261 patients from the ICH database between January 2015 and June 2018. There were 1106 patients with primary ICH, of whom 436 patients were within 24 hours of symptom onset and finally included. Of the 436 patients, 200 patients were within 6 hours of symptom onset. A flowchart depicting patient selection is shown in Figure 1.

The characteristics of included patients $(n=436)$ and excluded $(n=670)$ patients in our registry are shown in Table 1 . The patients included in this study were older $(\mathrm{P}<0.001)$, and had a higher prevalence of diabetes mellitus $(\mathrm{P}=0.026)$, worse baseline clinical severity scores (GCS and ICH score, all $\mathrm{P}<0.001$ ), higher antiplatelet medication use $(\mathrm{P}=0.008)$ and faster times from symptom onset to admission $\mathrm{CT}(\mathrm{P}<0.001)$. In addition, there were smaller baseline hematoma volumes $(\mathrm{P}<0.001)$, a lower prevalence of IVH $(\mathrm{P}=0.036)$ and a greater proportion of lobar ICH $(\mathrm{P}<0.001)$ in those with HE. Finally, the included patients had shorter PT $(\mathrm{P}=0.019)$ and lower INR $(\mathrm{P}=0.002)$

The included patients were predominately male (67.0\%) and had a mean age of 59.9 years ( \pm 14.0 years), with ages ranging from 20 to 94 years (Table 1). As expected, about $85 \%$ of patients had generally normal standard liver chemistry examination indices and coagulation indices; $12.6 \%$ had an AST $\geq 40 \mathrm{IU} / \mathrm{L}, 8.9 \%$ had an ALT $\geq 50$ IU/L, and $6.9 \%$ had an INR $\geq 1.15$. Moreover, the abnormal values of ALT, AST or INR were within two times the upper limit of the normal value (ULN). 
Association between liver fibrosis indices and substantial HE in patients within 24 hours of ICH onset

Of 436 patients within 24 hours of ICH onset, 115 (26.4\%) had substantial HE (Table 2). In general, patients with substantial HE were older (mean 63.0 vs 58.8 years, $\mathrm{P}=$ $0.007)$, tended to be male ( $74.8 \%$ vs $64.2 \%, \mathrm{P}=0.038)$, had larger baseline hematoma volumes (median $24.3 \mathrm{vs} 13.0 \mathrm{ml}, \mathrm{P}=0.005)$ and a lower proportion of deep ICH $(52.2 \%$ vs $70.7 \%, \mathrm{P}<0.001)$. The baseline GCS score, blood pressure on admission, the time from symptom onset to baseline CT and the time from baseline CT to follow-up CT scan were similar between groups (all $\mathrm{P}>0.05)$.

Patients with substantial HE showed significantly better values for AARPRI, FIB-4, mFIB-4, FibroQ and Forns index than those without substantial HE (all $p<0.1$ ), but there was no statistically significant difference in APRI $(p=0.245)$ between the two groups (Table 2). AARPRI, FIB-4, mFIB-4, FibroQ and Forns index with $p \leqslant 0.1$ were included in subsequent logistic regression.

The binary logistic regression Model 1, which adjusted for age, sex, hematoma location, and time from symptom onset to baseline CT scan, showed significant associations between substantial HE and each of the following indices: AARPRI [odds ratio (OR) $1.29,95 \%$ confidence interval (CI) 1.03-1.60], FIB-4 (OR 1.16, 95\% CI 1.03-1.30) and mFIB-4 (OR 1.05, 95\% CI 1.00-1.10). In contrast, substantial HE was not significantly 
associated with FibroQ or the Forns index (Table 3). In Model 2, which additionally considered the baseline hematoma volume, substantial HE was significantly associated only with AARPRI (OR 1.26, 95\% CI 1.00-1.57) and FIB-4 (OR 1.15, 95\% CI 1.02$1.30)$.

Association between liver fibrosis indices and substantial HE in patients within 6 hours of ICH onset

Of patients within 6 hours of symptom onset $(n=200), 60(30.0 \%)$ had substantial HE (Supplementary Table 1). For univariate analysis, the results were similar to those from patients admitted within 24 hours of onset, except age $(\mathrm{P}=0.096)$.

All six liver fibrosis indices, including APRI, AARPRI, FIB-4, mFIB-4, FibroQ and Forns index were associated with substantial HE in the univariate analysis and included in subsequent logistic regression (all $\mathrm{p}<0.05$, Supplementary Table 1). After adjusting the following variables in the binary logistic regression Model 1: age, sex and hematoma location, all six liver fibrosis indices were associated with substantial HE: APRI (OR 2.63, 95\% CI 1.30-5,34), AARPRI (OR 1.53, 95\% CI 1.08-2.16), FIB-4 (OR 1.34, 95\% CI 1.07-1.67), mFIB-4 (OR 1.08, 95\% CI 1.00-1.17), FibroQ (OR 1.08, 95\% CI 1.00-1.16) and Forns index (OR 1.37, 95\% CI 1.10-1.69). In Model 2, after considering the baseline hematoma volume, substantial HE was still significantly associated with all six liver fibrosis indices (all $\mathrm{P}<0.05$, Table 4). 


\section{Discussion}

In a Chinese population with a largely normal range of liver chemistry, we explored the association between various liver fibrosis and substantial HE after primary ICH. We found that at least two single liver fibrosis indices were independently associated with increased risk for substantial HE. Among primary ICH patients within 24 hours of symptom onset, AARPRI and FIB-4 were associated with substantial HE. While in patients within 6 hours of symptom onset, all six liver fibrosis indices were independently associated with substantial HE.

Growing evidence suggests an association between subclinical liver disease, such as liver fibrosis, and cerebrovascular disease. For example, the presence of liver fibrosis assessed with transient elastography is a strong predictor of long-term, all-cause mortality in the ischemic stroke population as well as a predictor of risk of ischemic stroke. $^{26,27}$ Another study showed liver fibrosis, as measured using FIB-4, to be independently associated with risk of hemorrhagic transformation in acute ischemic stroke patients. ${ }^{28}$ Consistent with these findings, our study indicates an association between liver fibrosis indices and substantial HE among ICH patients with largely normal standard liver chemistry. Our findings are consistent with a retrospective analysis of the Virtual International Stroke Trials Archive ICH database linking liver fibrosis (as assessed using two serum-based liver fibrosis indices) to admission hematoma volume, $\mathrm{HE}$ and 3-month mortality in Caucasians with normal liver chemistry. ${ }^{22}$ In addition, one study reported an association between subclinical 
alterations in individual hepatic enzymes, such AST and ALP, and worse clinical outcomes among ICH patients. ${ }^{12}$ This result was based on univariable analysis, and these associations were no longer significant after adjusting for confounders.

In our study, one in four patients experienced HE after primary $\mathrm{ICH}$, consistent with studies of HE incidence ranging from $13 \%$ to $38 \%$ in the United States, Japan and Italy. ${ }^{4,29}$ In our analysis, age, sex, hematoma location, baseline hematoma volume and platelet count were significantly associated with HE. Although the mechanisms underlying the association between liver fibrosis and HE remain obscure, some hypotheses can be proposed. First, the liver synthesizes numerous clotting factors involved in the coagulation cascade. Patients with liver disease may cause coagulation factor deficiency, leading to blood clotting abnormalities and increasing risk of developing HE. ${ }^{29}$ In addition, progression of liver fibrosis reduces the production of thrombopoietin and, hence, reduces platelet production. ${ }^{30}$ Second, vessel wall damage and vascular inflammation are associated with the development of fibrosis. ${ }^{31}$ In fact, one study suggested that subclinical coagulopathy, endothelial dysfunction, and vascular inflammation may be possible mechanisms between liver fibrosis and increased risk of HE. ${ }^{22}$ Further research should clarify how liver fibrosis affects risk of HE after ICH.

Our study has several limitations. First, it was a retrospective analysis based on data from a single center. Second, hematoma size was calculated using the $A * B * C / 2$ formula, 
while is widely use $\mathrm{d}^{24}$ but may not be as accurate as semi-automated measurement of hematoma size due to focusing on intraparenchymal HE assessment without IVH considered and not volumetrically measure. ${ }^{32}$ Third, liver fibrosis indices may be less accurate in patients without known liver diseases than more invasive, costlier methods such as liver biopsy and advanced imaging.

\section{Conclusions}

Despite the limitations, our study provides evidence that subclinical liver disease, as assessed with liver fibrosis indices, is related to risk of substantial HE after primary ICH in a Chinese cohort. These findings highlight the necessity of taking liver fibrosis into account routinely when screening ICH patients, for risk of substantial HE. Future cohort studies should investigate how liver fibrosis affects HE after ICH.

\section{Declarations}

\section{Acknowledgments}

We thank all staff members and participants involved in the study.

\section{Authors' contributions}

DW and SZ designed the study. JW, XY, JL, and ZH collected the data. HW and JL performed imaging analysis. HW and ZH performed statistics analysis. HW and JW drafted the main part of the manuscript. BW and SZ provided support for imaging 
analysis. SZ, DW, ML and BW helped design the study and revised the manuscript. All authors approved the final version submitted for publication.

\section{Funding}

Deren Wang was supported by the National Key Research and Development Program of China (grant no. 2018YFC1705006), the National Natural Science Foundation of China (grant no. 81870923), Chengdu Science and Technology Project (grant no. 2019GH02-00019-HZ), the 1·3.5 Project for Disciplines of Excellence-Clinical Research Incubation Project of West China Hospital (grant no. 2018HXFH041), and Sichuan University (grant no. 2018SCUH0066). Shihong Zhang was supported by the National Natural Science Foundation of China (grant no. 81974208) and the Key Application Research Program, Science \& Technology Department of Sichuan Province (grant no. 2018JY0101). Ming Liu was supported by the 1.3.5 Project for Disciplines of Excellence, West China Hospital, Sichuan University (grant no. ZYGD18009). Junfeng Liu was supported by the National Natural Science Foundation of China (grant no. 81901199).

\section{Availability of data and materials}

The datasets used and analysed during the current study are available from the corresponding author on reasonable request.

\section{Ethics approval and consent to participate}


The study was approved by the Ethics Committee on Biomedical Research, West China Hospital of Sichuan University (2019 362). No informed consent was required as this study was retrospective and all patient data were anonymized.

\section{Consent for publication}

Not applicable.

\section{Competing interests}

The authors declare that they have no competing interests.

\section{References}

1. Virani SS, Alonso A, Benjamin EJ, et al. Heart Disease and Stroke Statistics-2020 Update: A Report From the American Heart Association. Circulation 2020;141:e139-e596.

2. Gross BA, Jankowitz BT, Friedlander RM. Cerebral intraparenchymal hemorrhage: a review. JAMA 2019;321:1295-1303.

3. Schrag M, Kirshner H. Management of Intracerebral Hemorrhage: JACC Focus Seminar. J Am Coll Cardiol 2020;75:1819-1831.

4. Dowlatshahi D, Demchuk AM, Flaherty ML, Ali M, Lyden PL, Smith EE. Defining hematoma expansion in intracerebral hemorrhage: relationship with patient outcomes. Neurology 2011;76:1238-1244.

5. Delcourt $\mathrm{C}$, Huang $\mathrm{Y}$, Arima $\mathrm{H}$, et al. Hematoma growth and outcomes in intracerebral hemorrhage: the INTERACT1 study. Neurology 2012;79:314-319.

6. Roh D, Martin A, Sun CH, et al. ABO blood type and hematoma expansion after intracerebral hemorrhage: an exploratory analysis. Neurocrit Care 2019;31:66-71.

7. Mayer SA, Brun NC, Begtrup K, et al. Efficacy and safety of recombinant activated factor VII for acute intracerebral hemorrhage. N Engl J Med 2008;358: 
8. Baharoglu MI, Cordonnier C, Al-Shahi Salman R, et al. Platelet transfusion versus standard care after acute stroke due to spontaneous cerebral hemorrhage associated with antiplatelet therapy (PATCH): a randomized, open-label, phase 3 trial. Lancet 2016;387:2605-2613.

9. Hemphill JC 3rd, Greenberg SM, Anderson CS, et al. Guidelines for the Management of Spontaneous Intracerebral Hemorrhage: A Guideline for Healthcare Professionals From the American Heart Association/American Stroke Association. Stroke 2015;46:2032-2060.

10. Niizuma H, Shimizu Y, Nakasato N, Jokura H, Suzuki J. Influence of liver dysfunction on volume of putaminal hemorrhage. Stroke 1988:987-990.

11. Parikh NS, Merkler AE, Schneider Y, Navi BB, Kamel H. Discharge disposition after stroke in patients with liver disease. Stroke 2017;48:476-478.

12. Tan G, Hao Z, Lei C, Chen Y, Yuan R, Xu M, Liu M. Subclinical change of liver function could also provide a clue on prognosis for patients with spontaneous intracerebral hemorrhage. Neurol Sci 2016;37:1693-1700.

13. Caballería L, Pera G, Arteaga I, et al. High prevalence of liver fibrosis among European adults with unknown liver disease: a population-based study. Clin Gastroenterol Hepatol 2018;16:1138-1145.e5.

14. Watt GP, Lee M, Pan JJ, et al. High prevalence of hepatic fibrosis, measured by elastography, in a population-based study of Mexican Americans. Clin Gastroenterol Hepatol 2019; 17:968-975.e5.

15. Nallagangula KS, Nagaraj SK, Venkataswamy L, Chandrappa M. Liver fibrosis: a compilation on the biomarkers status and their significance during disease progression. Future Sci OA 2017;4:FSO250.

16. Mao X, Liu Z, Shi O, Yu K, Jiang Y, Jin L, Zhang T, Chen X. Non-invasive fibrosis markers are associated with mortality risk in both general populations and non-alcoholic fatty liver disease patients. Hepatol Res 2021 Jan;51:90-101.

17. Wang HW, Peng CY, Lai HC, et al. New noninvasive index for predicting liver fibrosis in Asian patients with chronic viral hepatitis. Sci Rep 2017;7:3259.

18. Ballestri S, Mantovani A, Baldelli E, et al. Liver fibrosis biomarkers accurately exclude advanced fibrosis and are associated with higher cardiovascular risk scores in patients with NAFLD or viral chronic liver disease. Diagnostics (Basel) 
2021;11:98.

19. Peleg N, Issachar A, Sneh-Arbib O, Shlomai A. AST to platelet ratio index and fibrosis 4 calculator scores for non-invasive assessment of hepatic fibrosis in patients with non-alcoholic fatty liver disease. Dig Liver Dis 2017;49:1133-1138.

20. Moreno C, Mueller S, Szabo G. Non-invasive diagnosis and biomarkers in alcohol-related liver disease. J Hepatol 2019;70:273-283.

21. Hsieh YY, Tung SY, Lee IL, et al. FibroQ: an easy and useful noninvasive test for predicting liver fibrosis in patients with chronic viral hepatitis. Chang Gung Med J 2009;32:614-622.

22. Parikh NS, Kamel H, Navi BB, et al. Liver fibrosis indices and outcomes after primary intracerebral hemorrhage. Stroke 2020;51:830-837.

23. Wang Q, Lin S, Dong W, Liu M. Characteristics of etiological diagnostic workup across the past 10 years in patients with spontaneous intracerebral hemorrhage in a large general hospital. Dev Neurorehabil 2016;19:10-16.

24. Kothari RU, Brott T, Broderick JP, et al. The ABCs of measuring intracerebral hemorrhage volumes. Stroke 1996;27:1304-1305.

25. Davis SM, Broderick J, Hennerici M, et al. Hematoma growth is a determinant of mortality and poor outcome after intracerebral hemorrhage. Neurology 2006;66: 1175-1181.

26. Baik M, Kim SU, Kang S, et al. Liver Fibrosis, Not Steatosis, Associates with long-term outcomes in ischaemic stroke patients. Cerebrovasc Dis 2019;47:3239.

27. Kim SU, Song D, Heo JH, et al. Liver fibrosis assessed with transient elastography is an independent risk factor for ischemic stroke. Atherosclerosis 2017;260:156-162.

28. Yuan CX, Ruan YT, Zeng YY, et al. Liver Fibrosis Is Associated with hemorrhagic transformation in patients with acute ischemic stroke. Front Neurol 2020;11:867.

29. Kazui S, Naritomi H, Yamamoto H, et al. Enlargement of spontaneous intracerebral hemorrhage. Incidence and time course. Stroke 1996;27:1783-1787.

30. Murthy SB, Moradiya Y, Dawson J, et al. Perihematomal edema and functional outcomes in intracerebral hemorrhage: Influence of hematoma volume and 
location. Stroke 2015;46:3088-3092.

31. Li Z, You M, Long C, et al. Hematoma Expansion in Intracerebral Hemorrhage: An Update on Prediction and Treatment. Front Neurol 2020;11:702.

32. Witsch J, Bruce E, Meyers E, et al. Intraventricular hemorrhage expansion in patients with spontaneous intracerebral hemorrhage. Neurology 2015;84:989-994. 


\section{Figures}

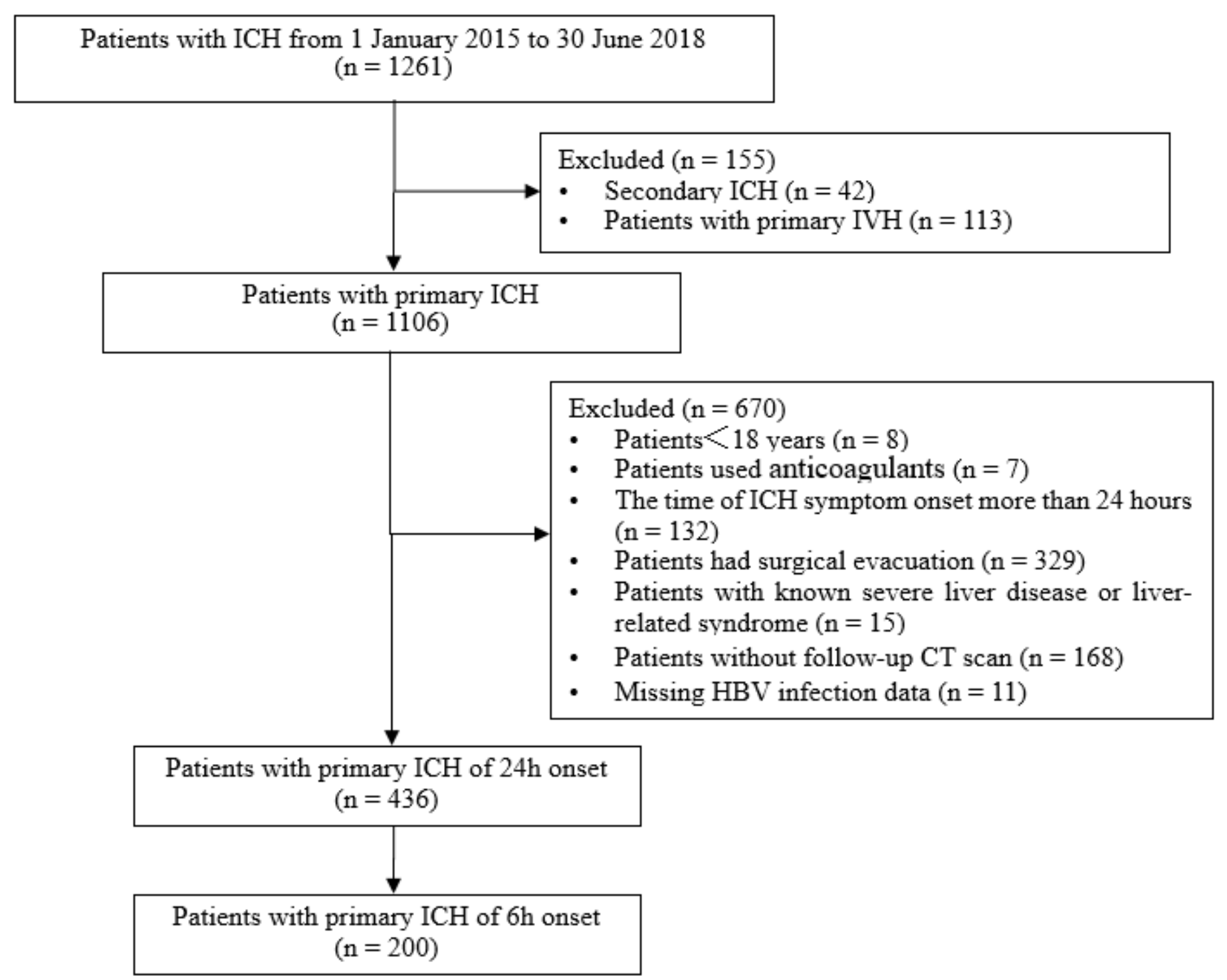

Figure 1

Selection of the study cohort

\section{Supplementary Files}

This is a list of supplementary files associated with this preprint. Click to download.

- Supplementarymaterial.docx 